\title{
The Normal Ordering Procedure and Coherent State of the Q-Deformed Generalized Heisenberg Algebra
}

\section{Won Sang Chung*}

Department of Physics and Research Institute of Natural Science, College of Natural Science, Gyeongsang National University, Jinju 660-701, Korea

\author{
Abstract \\ In this paper we discuss the normal ordering procedure of the q-deformed generalized Heisenberg algebra. We \\ also obtain the coherent state for some types of characteristic functions.
}

Keywords: Heisenberg algebra; q-deformed

\section{Introduction}

In the last few years quantum algebras and quantum groups have been the subject of intensive research in several physics and mathematics fields. Quantum groups or q-deformed Lie algebra implies some specific deformation of classical Lie algebra. From the mathematical point of view, it is a non-commutative associative Hopf algebra. The structure and representation theory of quantum groups have been developed extensively by Jimbo [1] and Drinfeld [2]. In the study of the basic hypergeometric function Jackson [3] invented the Jackson derivative and integral, which is now called q-derivative and q-integral. Jackson's pioneering research enabled theoretical physicists and mathematician to study the new physics or mathematics related to the q-calculus. Much was accomplished in this direction and work is under way to find the meaning of the deformed theory. By using the q- calculus, Arik and Coon [4] proposed the q-deformation of the Heisenberg algebra as follows;

$$
\begin{aligned}
& a a^{\dagger}-q a^{\dagger} a=1 \\
& {\left[N, a^{\dagger}\right]=a^{\dagger},[N, a]=-a}
\end{aligned}
$$

Where $N=N^{\dagger}$ is called a number operator and $a=\left(a^{\dagger}\right)^{\dagger}$ Following the approach of the authors of ref [4], several deformed Heisenberg algebra has been proposed in the literature [5-10]. In most of deformed Heisenberg algebra, authors adopted the same commutation relations between the number operator and step operators and deformed the commutation relation between $a$ and $a^{\dagger}$

In 2000, the new generalization of the Heisenberg algebra was introduced by Rego-Monteiro and Curado [11-13], which takes the form

$$
H a^{\dagger}=a^{\dagger} f(H), a H=F(H) a,\left[a, a^{\dagger}\right]=f(H)-H
$$

where $H$ is a hamiltonian of the physical system under consideration and $f(H)$ is an analytic function of $H$, called a characteristic function of the algebra. In this algebra, the commutation relations between the number operator and step operators were changed into the more general form which is characterized in terms of the function of the number operator. The authors of the ref $[11,12]$ called this function a characteristic function and discussed the cases when the characteristic function is linear and quadratic in the number operator [12].

In this paper we change the algebra (2) by introducing the parameter $\mathrm{q}$ and discuss the normal ordering procedure of the q-deformed generalized Heisenberg algebra (GHA). We use some operator identities to construct the q-deformed generalized Stirling operator of the second kind and its generating function. We also present the q-deformed generalized Heisenberg algebra whose characteristic function is a MÄobius transformation. Finally we discuss some characteristic functions giving a Klauder's coherent state.

\section{Q Deformed Generalized Heisenberg Algebra}

In this section we discuss the representation theory of the q-deformed generalized Heisenberg algebra. The q-deformed generalized Heisenberg algebra takes the following form;

$$
H a^{\dagger}=a^{\dagger} f(H), a H=f(H) a,\left[a, a^{\dagger}\right]_{q}=a a^{\dagger}-q a^{\dagger} a=f(H)-q H,
$$

Where $H$ is a hamiltonian of the physical system under consideration and $f(H)$ is an analytic function of $H$, called a characteristic function of the algebra. The deformation parameter is related to the concrete form of $f(H)$ and a large class of type Heisenberg algebra can be obtained by choosing the function $f(H)$. From now on we restrict our concern to the case of

$$
0<q<1
$$

For example, if we take $f(H)=1+q H$, the algebra (3) reduces to the q-deformed Heisenberg algebra where the hamiltonian is related to the number operator $N$ as follows

$$
H=[N]=\frac{1-q^{N}}{1-q}
$$

The choice of $f(H)$ gives a lot of deformed algebra, which is the reason why $f(H)$ is called a characteristic function of the algebra. Now let us introduce the q-Casimir operator as follows;

$$
C q=a^{\dagger} a-f(H)=q^{-1}\left(a a^{\dagger}-f(H)\right), C_{q}^{\dagger}=C q
$$

We demand that the q-Casimir operator obeys

$$
[C q, H]=0,\left[a, C_{q}\right]_{q}=0,\left[C_{q}, a^{\dagger}\right]_{q}=0
$$

When $q$ goes to a unity, the q-Casimir operator reduces to an ordinary Casimir operator. If

*Corresponding author: Won Sang Chung Department of Physics and Research Institute of Natural Science, College of Natural Science, Gyeongsang National University, Jinju 660-701, Korea, E-mail: mimip4444@hanmail.net

Received December 05, 2013; Accepted October 24, 2014; Published Octobe 31,2014

Citation: Chung WS (2014) The Normal Ordering Procedure and Coherent State of the Q-Deformed Generalized Heisenberg Algebra. J Generalized Lie Theory Appl 8: 213. doi: 10.4172/1736-4337.1000213

Copyright: () 2014 Chung WS. This is an open-access article distributed unde the terms of the Creative Commons Attribution License, which permits unrestricted use, distribution, and reproduction in any medium, provided the original author and source are credited. 


$$
\begin{aligned}
& \text { We let } \mathrm{C}=\mathrm{C}_{\mathrm{q}} \mathrm{C}_{\mathrm{q}-1} \text {, we have } \\
& {[C, H]=\left[C, a^{\dagger}\right]=[C, a]=0}
\end{aligned}
$$

Now let us construct the irreducible representation of the algebra (3) by introducing the ground state $|0\rangle$ with the lowest eigenvalue of $H$ obeying

$$
H|0\rangle=\epsilon_{0}|0\rangle
$$

Let $|n\rangle$ be a normalized eigenstate of $H$

$H|n\rangle=\epsilon_{n}|n\rangle, \quad n=0,1,2, \ldots$

Applying $H$ on $a^{\dagger}|n\rangle$ yields

$$
H\left(a^{\dagger}|n\rangle\right)=a^{\dagger} f(H)|n\rangle=f\left(\epsilon_{n}\right)\left(a^{\dagger}|n\rangle\right),
$$

Which means that $a^{\dagger}|n\rangle$ is an eigen state of $H$ with eigen value $f\left(\epsilon_{n}\right)$ Applying $a^{\dagger}$ on the ground state successively, we have

$$
H\left(\left(a^{\dagger}\right)^{n}|0\rangle\right)=f^{n}\left(\epsilon_{0}\right)\left(\left(a^{\dagger}\right)^{n}|0\rangle\right),
$$

Where $\epsilon_{n}=f^{n}\left(\epsilon_{0}\right)$ denotes the $\mathrm{n}$-th iterate of $f$ defined as $f^{n}\left(\epsilon_{0}\right)=\left(f\left(f\left(f\left(\ldots f\left(\epsilon_{0}\right)\right)\right)\right)\right.$ If we assume that $\left(a^{\dagger}\right)^{n}|0\rangle$ is proportional to $|n\rangle$, we have

$\epsilon_{n}=f^{n}\left(\epsilon_{0}\right)=f\left(\epsilon_{n-1}\right)$

So all eigen values of $H$ are determined from $\epsilon_{0}$ through $f$.

Acting $a$ on $|n+1\rangle$ we get

$a(H|n+1\rangle)=f(H)(a|n+1\rangle)=\epsilon_{n-1}(a|n+1\rangle)=f\left(\epsilon_{n}\right)(a|n+1\rangle)$,

We get

$$
H(a|n+1\rangle)=\epsilon_{n}(a|n+1\rangle),
$$

Which shows that $a|n+1\rangle$ is also an eigen state of $H$ with eigen value $\epsilon_{n}$ and $a|n+1\rangle$ is proportional to $|n\rangle$

The representation of the q-deformed generalized Heisenberg algebra is then given by

$$
\begin{aligned}
& H|n\rangle=\epsilon_{n}|n\rangle, n=0,1,2,3 \ldots \\
& a^{\dagger}|n\rangle=N_{n}|n+1\rangle, a|n\rangle=N_{n-1}|n-1\rangle,
\end{aligned}
$$

Where

$$
N_{n}^{2}=f^{n+1}(\epsilon)-q^{n+1} \epsilon_{0}=\epsilon_{n+1}-q^{n+1} \epsilon_{0}, \quad N_{-1}=0
$$

The relation between step operators and hamiltonian is given by

$$
\begin{aligned}
& a a^{\dagger}=f(H)-\epsilon_{0} \mathrm{P}_{q} \\
& a^{\dagger} a=H-q^{-1} \epsilon_{0} \mathrm{P}_{q},
\end{aligned}
$$

Where we have

$$
\mathrm{P}_{q}|n\rangle=q^{n}|n\rangle
$$

Here $P q$ is a q-projection operator satisfying

$$
a P_{q}=q P_{q} a, P q a^{\dagger}=q a^{\dagger} P_{q}, \quad P_{q} H=H P_{q}
$$

Then we have

$$
|n\rangle=\frac{1}{N_{0}^{n} \sqrt{[n]_{f} !}}\left(a^{\dagger}\right)^{n}|0\rangle,
$$

Where f-number is defined as

$$
[n]_{f}=\frac{N_{n-1}^{2}}{N_{0}^{2}}=\frac{f^{n}\left(\epsilon_{0}\right)-q^{n} \epsilon_{0},}{f\left(\epsilon_{0}\right)-q \epsilon_{0}}
$$

And

$$
N_{0}^{2}=f\left(\epsilon_{0}\right)-q \epsilon_{0}
$$

The $(n+1)$-th eigenvalue $e_{n+1}$ of the Hamiltonian depends on the previous eigenvalue $e_{n}$

$$
e_{n+1}=f\left(e_{n}\right)
$$

So this algebra is sometimes called a one step algebra.

The representation can be rewritten in terms of the f-number as follows;

$$
a^{\dagger}|n\rangle=N_{0} \sqrt{[n+1]_{f}}|n+1\rangle, a|n\rangle=N_{0} \sqrt{[n]_{f}}|n-1\rangle,
$$

\section{Normal Ordering Process and F-stirling Operator}

Now we discuss the normal ordering process for the q-deformed generalized Heisenberg algebra. From the second relation of the eq.(3), we have

$$
a \Phi(H)=\Phi(f(H)) a
$$

For an arbitrary function $\Phi(H)$. For $\Phi(x)=f(x)$, we get

$a^{k} f(H)=f^{k+1}(\mathrm{H}) \mathrm{a}^{\mathrm{k}}, f(H)\left(a^{\dagger}\right)^{k}=\left(a^{\dagger}\right)^{k} f^{k+1}(H)$

Replacing $H \rightarrow f^{-1}(H)$ in the first relation of the eq.(3), we have

$a^{\dagger} H=f^{-1}(H) a^{\dagger}$

or generally

$\left(a^{\dagger}\right)^{k} H=f^{-k}(H) a^{\dagger}$

And

$$
H a^{k}=a f^{-k}(H)
$$

Where

$$
f^{-k}=\underbrace{f^{-1} \circ f^{-1} \circ \cdots \circ f^{-1}}_{k}
$$

Then we have the following formulas;

$$
\begin{aligned}
a^{k}\left(a^{\dagger}\right)^{k} & =\prod_{j=1}^{k}\left[f^{j}(H)-q^{j-1} \epsilon_{0} P_{q}\right] \\
\left(a^{\dagger}\right)^{k} a^{k} & =\prod_{j=0}^{k-1}\left[f^{-j}(H)-q^{-(j+1)} \epsilon_{0} P_{q}\right],
\end{aligned}
$$

Where $f^{0}(H)=f(H)$.

The f-Stirling operator of the second kind is defined as 


$$
\left(a^{\dagger} a\right)^{n}=\sum_{k=1}^{n}\left(a^{\dagger}\right)^{k} S(n, k, H) a^{k}
$$

Where $S(n ; k ; H)$ is the $\mathrm{f}$-Stirling operator of the second kind. Using $\left(a^{\dagger} a\right)^{n+1}=\left(a^{\dagger} a\right)\left(a^{\dagger} a\right)^{n}$,

We can obtain the recurrence relation

$$
\begin{aligned}
S(n+1,1, H) & =(f(H)-q H) S(n, 1, H), \\
S(n+1, k, H)= & q^{k-1} S(N, K-1, f(H))=\left(f^{k}(H)-q^{k} H\right) S(n, k, H),(1 \leq k \leq n) \\
& S(n+1, n+1, H)=q^{n} S(n+1, n, f(H)),
\end{aligned}
$$

Where $S(I, j, H)=0$ for $i<j$ and we used the following formulas

$$
\begin{gathered}
a\left(a^{\dagger}\right)^{k}=q^{k}\left(a^{\dagger}\right)^{k} a+\left(a^{\dagger}\right)^{k-1}\left(f^{k}(H)-q^{k} H\right) \\
a^{k} a^{\dagger}=q^{k} a^{\dagger} a^{k}+\left(f^{k}(H)-q^{k} H\right) a^{k-1}
\end{gathered}
$$

The first few Stirling operator of the second kind are

$$
\begin{gathered}
S(1,1, H)=I, \\
S(2,1, h)=f(H)-q H, S(2,2, H)=q, \\
S(3,1 H)=f(H)-q H)^{2}, S(3,2, H)=2 q f^{2}(H)-q^{2} f(H)-q^{3} H, S(3,3, H)=q^{2} \\
S(4,1, H)=(f(H)-q H)^{3} \\
S(4,2, H)=3 q\left(f^{2}(H)\right)^{2}-3 q^{2} f^{2}(H) f(H)+q^{3}(f(H))^{2}-3 q^{3} H f^{2}(H)+q^{4} H f(H)+q^{5} H^{2}, \\
S(4,3, c)=q^{2}(2 q+1) f^{3}(H)-q^{4} f^{2}(H)-q^{5} f(H)-q^{5} H, S(4,4, H)=q^{3}
\end{gathered}
$$

We define the generating function of the f-Stirling operator of the second kind in the form

$$
P_{k}(H \mid x)=\sum_{n=k}^{\infty} S(n, k, H) x^{n}
$$

The recurrence relations are then given by

$$
\begin{aligned}
& P_{1}(H \mid x)=\frac{x}{1-(f(H)-q(H) x} \\
& P k(H \mid x)=\frac{q^{k-1} x}{1-\left(f^{k}(H)-q^{k} H\right) x} p_{k-1}(f(H) \mid x),(k>1)
\end{aligned}
$$

If we set $P_{0}(H \mid x)=I$, we get

$$
P_{k}(H \mid x)=\prod_{j=0}^{k-1} \frac{q^{k-1-j} x}{1-\left(f^{k}(H)-q^{k-j} f^{r}(H)\right) x}
$$

The eq.(42) can be written in terms of a sum of partial fractions

$$
P_{k}(H \mid x)=q^{\frac{k(k-1)}{2}} x^{k} \sum_{r=0}^{k-1} \frac{p_{r}}{\left.1-) f^{k}(H)-q^{k-r} f^{r}(H)\right) x},
$$

Where

$$
p_{r}=\frac{1}{\prod_{j=0, j \neq \mathrm{r}}^{k-1}\left(1-\frac{f^{k}(H)-q^{k-j} f^{j}(H)}{f^{k}(H)-q^{k-r} f^{r}(H)}\right)}
$$

Therefore the f-Stirling operator of the second kind takes the following form;

$$
S(n, k, H)=q^{\frac{k(k-1)}{2}} \sum_{r=0}^{k-1} \frac{\left(f^{k}(H)-q^{k-r} f^{r}(H)\right)^{n-1}}{\prod_{j=1, j \neq r}^{k}\left(q^{k-j} f^{j}(H)-q^{k-r} f^{r}(H)\right)}
$$

\section{The Deformed Heisenberg Algebra Related to the Mäobius Transformation}

In this section we are going to find the representation for the algebra defined by the relation given in the eq.(3) considering

$$
f(H)=\frac{\gamma H+\delta}{\alpha H+\beta},
$$

Where $\alpha, \beta, \gamma, \delta$ are real. The ordinary Heisenberg algebra and q-deformed Heisenberg algebra are obtained from the suitable choice of $\alpha, \beta, \gamma, \delta$ inverse of the MÄobius transformation is given by

$$
f^{-1}(H)=\frac{\beta H-\delta}{-\alpha H+\gamma}
$$

Where $\beta \gamma-\alpha \delta \neq 0$.

In the choice of the characteristic function given in the eq.(46), the algebra (3) takes the following form;

$$
\begin{aligned}
& \beta H a^{\dagger}-\gamma a^{\dagger} H=\delta a^{\dagger}-\alpha H a^{\dagger} H, \\
& \beta a H-\gamma H a=\delta a-\alpha H a H, \\
& (\alpha H+\beta) a a^{\dagger}-q(\alpha H+\beta) a^{\dagger} a=\delta+(\gamma-q \beta) H-q \alpha H^{2}
\end{aligned}
$$

Now let us introduce the following characteristic function;

$$
f(H)=\frac{\gamma H}{1-\alpha H}
$$

Where we assume that $\alpha>0 ; 0<\gamma<1$. Then we have the following algebra

$$
\begin{aligned}
& H a^{\dagger}-\gamma a^{\dagger} H=\alpha H a^{\dagger} H, \\
& a H-\gamma H a=\alpha H a H, \\
& (1-\alpha H) a a^{\dagger}-q(1-\alpha H) a^{\dagger} a=(\gamma-q) H+\alpha q H^{2}
\end{aligned}
$$

The inverse of the characteristic function is given by

$$
f^{-1}(H)=\frac{H}{\gamma+\alpha H}
$$

The $\mathrm{n}$-the iterate of $f$ is then given by

$$
f^{n}(H)=\frac{\gamma^{n} H}{1-\alpha[n]_{\gamma} H}
$$

Where $[n]_{\gamma}=\frac{1-\gamma^{n}}{1-\gamma}$

\section{Representation}

For the characteristic function given in the eq.(49), we have

$$
\begin{aligned}
& N_{0}^{2}=\frac{\epsilon_{0}\left(\gamma-q+\alpha q \epsilon_{0}\right)}{1-\alpha \epsilon_{0}} \\
& {[n]_{f}=\frac{\left(1-\alpha \epsilon_{0}\right)\left(\gamma^{n}-q^{n}+\alpha \epsilon_{0} q^{n}[n]_{\gamma}\right)}{\left(\gamma-q+\alpha q \epsilon_{0}\right)\left(1-\alpha[n] \gamma \epsilon_{0}\right)}}
\end{aligned}
$$

From $N_{0}^{2}>0,[n]_{f}>0$ for all $n$, we have

$\frac{1}{\alpha}\left(1-\frac{\gamma}{q}\right) \leq{ }_{0}^{o} \leq \frac{1}{\alpha}(1-\gamma)$ 
Where we assumed that

$\epsilon_{0}>0, \alpha>0,0<\gamma<1, q>\gamma$

The representation takes the following form;

$$
\begin{aligned}
& H|n\rangle=\left(\frac{\gamma^{n} \epsilon_{0}}{1-\alpha[n]_{\gamma} \epsilon_{0}}\right)|n\rangle \\
& a|n\rangle=\sqrt{\frac{\epsilon_{0}\left(\gamma^{n}-q^{n}+\alpha \epsilon_{0} q^{n}[n]_{\gamma}\right.}{1-\alpha \epsilon_{0}[n]_{\gamma}}}|n-1\rangle \\
& a^{\dagger}|n\rangle=\sqrt{\frac{\epsilon_{0}\left(\gamma^{n+1}-q^{n+1}+\alpha \epsilon_{0} q^{n+1}[n+1]_{\gamma}\right.}{1-\alpha \epsilon_{0}[n+1]_{\gamma}}}|n+1\rangle
\end{aligned}
$$

\section{Coherent state}

We define a coherent state as a eigenvector of the annihilation operator as follows:

$$
a|z\rangle=z|z\rangle,
$$

Where $z$ is a complex number. The coherent state can be represented by using the number state as follows :

$$
|z\rangle=\sum_{n=0}^{\infty} c_{n}(z)|n\rangle
$$

Inserting the eq.(58) into the eq.(57), we obtain the following relations :

$$
c_{n}(z)=\sqrt{\frac{1-\alpha \epsilon_{0}[n]_{\gamma}}{\gamma^{n}-q^{n}+\alpha \epsilon_{0} q^{n}[n]_{\gamma}}} z c_{n-1}(z)
$$

Solving the eq.(59), the coherent state is given by

$$
z\rangle=c_{0}\left(|z|^{2} \sum_{n=0}^{\infty} \sqrt{\coprod_{k=1}^{n} \frac{1-\alpha \epsilon_{0}[k]_{\gamma}}{\gamma^{k}-q^{k}+\alpha \epsilon_{0} q^{k}[k]_{\gamma}}}\left(\frac{z}{\sqrt{\epsilon_{0}}}\right)^{n},\right.
$$

Where

$$
c_{0}\left(|z|^{2}\right)=\left[e_{q, \alpha, \gamma}\left(\frac{|z|^{2}}{\epsilon_{0}}\right)\right]^{-1 / 2}
$$

And

$$
e_{q, \alpha, \gamma}(x)=\sum_{n=0}^{\infty} \sqrt{\prod_{k=1}^{n} \frac{1-\alpha \epsilon_{0}[k]_{\gamma}}{\gamma^{k}-q^{k}=\alpha \epsilon_{0} q^{k}[k]_{\gamma}}} x^{n}
$$

\section{Some Characteristic Functions giving Klauder's Coherent State}

In this section we discuss the some characteristic functions giving q-deformed Klauder's coherent state (KCS). The KCS should satisfy the normalizability

$$
\langle z \mid z\rangle=1
$$

and the completeness

$$
\int d_{q}^{2} z w(|z|)|z\rangle\langle z|=1,
$$

Where we use the q-integral instead of the ordinary integral and assume $0 \leq|z|^{2}<1$.

From the definition of the coherent state

$$
a|z\rangle=z|z\rangle
$$

We have

$$
|z\rangle=c_{0}\left(|z|^{2}\right) \sum_{n=0}^{\infty} \frac{z^{n}}{N_{n-1} !}|n\rangle
$$

The case of $f(x)=\frac{1}{[2]-q x}$

In this choice we have

$$
f^{n}(x)=\frac{[n]-q[n-1] x}{[n+1]-q[n] x},
$$

Where we used

$$
[2][n+1]-q[n]=[n+2]
$$

If we choose $\epsilon_{0}=0$ we have

$\epsilon_{n}=N_{n-1}^{2}=\frac{[n]}{[n+1]}$

The coherent state is then given by

$$
|z\rangle=c_{0}\left(|z|^{2}\right) \sum_{n=0}^{\infty} \sqrt{[n+1] z^{n}|n\rangle},
$$

Where

$c_{0}^{2}(x)=(1-x)_{q}^{2}, \quad x=|z|^{2}$

And

$$
(1-a)_{q}^{n}=\prod_{k=0}^{n-1}\left(1-q^{k} a\right)
$$

Now we have to find the weighting function $w(x)$ so that the coherent sate may satisfy the Completeness. If we set $z=r e^{i \theta}, x=r^{2}$ and assume $d_{q}^{2} z=r d_{q} r d \theta$, we have

$$
\pi \sum_{n=0}^{\infty}|n\rangle|n\rangle \int_{0}^{1} d_{q} x w(x)(1-x)_{q}^{2}[n+1] x^{n}=1
$$

We can easily find the weighting function

$$
w(x)=\frac{1}{\pi(1-x)_{q}^{2}},
$$

Where we used

$$
\int_{0}^{1} d_{q} x x^{n}=\frac{1}{[n+1]}
$$

The case of $f(x)=\left(\frac{1}{[2]-q \sqrt{x}}\right)^{2}$

In this choice we have

$$
f^{n}(x)=\left(\frac{[n]-q[n-1] \sqrt{x}}{[n+1]-q[n] \sqrt{x}}\right)^{2}
$$


If we choose $\epsilon_{0}=0$ we have

$\epsilon_{n}=N_{n-1}^{2}=\left(\frac{[n]}{[n+1]}\right)^{2}$

The coherent state is then given by

$$
|z\rangle=c_{0}\left(|z|^{2}\right) \sum_{n=0}^{\infty}[n+1] z^{n}|n\rangle
$$

Where

$$
c_{0}^{2}(x)=\frac{(1-x)_{q}^{3}}{1+q x}
$$

Now we have to find the weighting function $w(x)$ so that the coherent sate may satisfy the completeness, which implies

$$
\pi \sum_{n=0}^{\infty}|n\rangle\langle n| \int_{0}^{1} d_{q} x w(x) c_{0}^{2}(x)[n+1]^{2} x^{n}=1
$$

We can easily find the weighting function

$$
w(x)=\frac{(1-q)(1+q x) \ln (q x)}{\pi \ln q(1-x)_{q}^{3}},
$$

Where we used

$$
\int d x x \ln (q x) \frac{\ln }{(1-q)[n+1]}
$$

The case of $f(x)=\left(\frac{[n]-q[n-1] x^{1 / p}}{[n+1]-q[n] x^{1 / p}}\right) p=1 ; 2 ; 3$;

In this choice we have

$$
f^{n}(x)=\left(\frac{[n]-q[n-1] x^{1 / p}}{[n+1]-q[n] x^{1 / p}}\right)^{p}
$$

If we choose $\epsilon_{0}=0$ we have

$\epsilon_{n}=N_{n-1}^{2}=\left(\frac{[n]}{[n+1]}\right)^{p}$

The coherent state is then given by

$|z\rangle=c_{0}\left(|z|^{2}\right) \sum_{n=0}^{\infty}[n+1]^{p / 2} z^{n}|n\rangle$

Where

$$
c_{0}^{2}(x)=\frac{x}{\operatorname{li}_{-p}^{q}(x)}
$$

and the q-polylogarithm function is defined by

$$
\operatorname{li}_{-p}^{q}(x)=\sum_{n=0}^{\infty}[n+1]^{p} x^{n+1}
$$

Now we have to find the weighting function $w(x)$ so that the coherent sate may satisfy the completeness, which implies

$$
\pi \sum_{n=0}^{\infty}|n\rangle\langle n| \int_{0}^{1} d_{q} x w(x) c_{0}^{2}(x)[n+1]^{p} x^{n}=1
$$

The eq.(70) is rewritten as $\int_{0}^{1} d_{q} x x^{n}=\frac{q-1}{q^{n+1}-1}$

If we set $a=q^{n+1}$, we get $\frac{\partial}{\partial_{n}}=(\ln q) a \frac{\partial}{\partial a}$

Differentiating both sides of the eq.(89) $p$ times with respect to $n$, we have

$\int_{0}^{1} d_{q} x x^{n}(\ln x)^{p}=(1-q)(\ln q)^{p}\left(a \frac{\partial}{\partial a}\right)^{p}\left(\frac{1}{1-a}\right)$

Where

$\left(a \frac{\partial}{\partial a}\right)^{p}\left(\frac{1}{1-a}\right)=\left(a \frac{\partial}{\partial a}\right)^{p} \sum_{k=0}^{\infty} a^{k}=\sum_{k=0}^{\infty} k^{p} a^{k}$

If we set

$\left(a \frac{\partial}{\partial a}\right)^{p}\left(\frac{1}{1-a}\right)=\frac{a}{(1-a)^{p+1}}=\sum_{k=0}^{p-1} c_{k}^{(p)} a^{k}$,

We have the following recurrence relation

$(k+1) \mathrm{c}_{k}^{(p)}+(p+1-k) c_{k-1}^{(p)}=c_{k}^{(p+1)},(k=1,2, \ldots, N-1)$

And

$c_{0}^{(p)}=c_{p-1}^{(p)}=1$

For $k=1$, the eq.(93) is as follows :

$c_{1}^{(p+1)}=2 c_{1}^{(p)}+p$

Solving the eq.(95), we have

$c_{1}^{(p)}=\sum_{k=1}^{p-1} k \cdot 2^{p-1-k}=2^{p}-(p+1), \quad(p \geq 2)$

For general $k$, we have

$c_{1}^{(p)}=\sum_{i=2}^{p}(i-k) c_{k-1}^{(i-1)}(k+1)^{p-i} \theta(i-k-1) \quad(p \geq 2)$

And

$c_{k}^{(1)}=0,(k \geq 1)$,

Where

$\theta(x)= \begin{cases}1 & (x \geq 0) \\ 0 & (x<0)\end{cases}$

Using the formula (92), we have

$\int_{0}^{1} d_{q} x x^{n}(\ln x)^{p}=\frac{(-1)^{p}(q-1)}{(a-1)^{p+1}} \sum_{k=0}^{p-1} c_{k}^{(p)} a^{k+1}$

If we expand the summation of the eq.(100) in terms of $a_{i} 1$, we get

$\sum_{k=0}^{p-1} c_{k}^{(p)} a^{k+1}=\sum_{i=0}^{p} B_{l}^{(p)}(a-1)^{l}$,

Where

$B_{l}^{(p)}=\frac{1}{l !} \sum_{k=0}^{p-1} k(k-1)(k-2) \ldots(k-(l-1)) c_{k}^{(p)}$ 
Therefore we have the following formula;

$$
\int_{0}^{1} d_{q} x x^{n}(\ln x)^{p}=(-1)^{p}(\ln q)^{p} \sum_{k=0}^{p} \frac{B_{k}^{(p)}(q-1)^{k-p}}{[n+1]^{p+1-k}}
$$

If we define the new function

$$
L_{P}(\mathrm{x})=\frac{(1-q)^{p}}{B_{0}^{(p)}(\ln q)^{p}}\left((\ln x)^{p}-(\ln q)^{p} \sum_{k=0}^{p-1}(1-q)^{k-p} B_{k}^{(p)} L_{p+1-k}(x)\right),
$$

We have

$$
\int_{0}^{1} d_{q} x x^{n} L_{p}(x)=\frac{1}{[n+1]^{p+1}}
$$

The first few $\mathrm{L} p(x)$ are as follows;

$$
\begin{aligned}
& L_{0}(x)=1 \\
& L_{1}(x)=\frac{1-q}{\ln q}(\ln x+\ln q) \\
& \mathrm{L}_{2}(x)=\frac{(1-q)^{2}}{4(\ln q)^{2}}\left((\ln x)^{2}-7(\ln q)(\ln x)-4(\ln q) 2\right)
\end{aligned}
$$

Therefore we obtain the weighting function as follows;

$$
w(x)=\frac{\operatorname{li}_{-p}^{q}(x) \mathrm{L}_{p}(x)}{\pi x}
$$

\section{Conclusion}

In this paper we discussed the normal ordering procedure of the q-deformed generalized Heisenberg algebra, where we introduced the q-deformed generalized Stirling operator of the second kind instead of the Stirling number of the second kind and constructed its generating function. We also discussed the q-deformed generalized Heisenberg algebra whose characteristic function is a MÄobius transformation. Finally we discussed some types of characteristic functions giving a Klauder's coherent state. In fact, it is possible to construct more general algebra as

$$
\begin{aligned}
& H a^{\dagger}=a^{\dagger} f(H), \quad a H=f(H) a, \\
& {\left[a, a^{\dagger}\right]_{q}=\prod_{k=0}^{d}(f(H)+k)-q \prod_{k=0}^{d}(H+k)}
\end{aligned}
$$

It is tempting to investigate, as we did in this paper, the above algebra for some interesting characteristic function. We hope that this topic and its related one will become clear in the near future.

\section{References}

1. Jimbo $M$ (1985) A $q$-analogue of $U(g[(N+1))$, Hecke algebra, and the YangBaxter equation Lett Math Phys 10: 63.

2. Jimbo $M(1986) A q$-difference analogue of $U(g)$ and the Yang-Baxter equation. Lett Math Phys 11: 247.

3. Drinfeld V (1986) Quantum Groups. Proc intern congress of Mathematicians Berkeley, California, USA.

4. F. Jackson, Mess. Math. 38, 57 (1909).

5. Arik M, Coon D (1976) Hilbert spaces of analytic functions and generalized coherent states. J Math Phys 17: 524

6. Macfarlane AJ (1989) On q-analogues of the quantum harmonic oscillator and

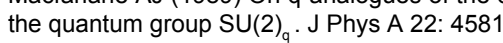

7. Biedenharn $L$ (1989) The quantum group $S_{\mathrm{q}}(2)$ and a q-analogue of the boson operators. J Phys A 22: L873

8. Chung W, Chung K, Nam S,UmC (1993) Generalized deformed algebra.Phys Lett $A, 183: 363$

9. Borozov E, Damaskinsky V, Yegorov S (1995) Some Remarks on the Representation of the Generalized Deformed Oscillator Algebra. q-alg/9509022.

10. Quesne C, Vansteenkiste N (1998) C $\lambda$-extended harmonic oscillator and (para) supersymmetric quantum mechanics. Phys Lett A 240: 21.

11. Daoud M, El Kinani EH (2011) Extended Weyl-Heisenberg algebra, phase operator, unitary depolarizers and generalized Bell states. Phys Lett A 375: 2492.

12. Curado E, Rego-Monteiro M (2000) Thermodynamic properties of a solid exhibiting the energy spectrum given by the logistic map.PhysRev E61: 6255.

13. Rego-Monteiro M, Curado E (2001) Multi-parametric deformed Heisenberg algebras: a route to complexity. J Phys A 34:3253.
Citation: Chung WS (2014) The Normal Ordering Procedure and Coherent State of the Q-Deformed Generalized Heisenberg Algebra. J Generalized Lie Theory Appl 8: 213. doi: 10.4172/1736-4337.1000213
Submit your next manuscript and get advantages of OMICS Group submissions

Unique features:

- User friendly/feasible website-translation of your paper to 50 world's leading languages Audio Version of published paper

Digital articles to share and explore

Special features:

- 35 Open Access Journals

30,000 editorial team

21 days rapid review process

21 days rapid review process
Quality and quick editorial, review and publication processing

Quality and quick editorial, review and publication processing

Indexing at PubMed (partial), Scopus, EBSCO,
Sharing Option: Social Networking Enabled

Sharing Option: Social Networking Enabled
Authors, Reviewers and Editors rewarded with online Scientific Credits

Better discount for your subsequent articles

Submit your manuscript at: http://www.omicsonline.org/submission 